REVUE FRANCOPHONE DE LA

DÉFICIENCE INTELLECTUELLE

VOLUME 23, 143-153

\title{
LA RÉSOLUTION DE PROBLÈMES DES PARENTS D'ENFANTS AYANT UNE DÉFICIENCE INTELLECTUELLE
}

\author{
Natasha Tremblay, Lise Lachance, Alain Côté et Louis Richer
}

\begin{abstract}
Les études concernant le stress et la santé psychologique des parents d'enfants ayant une déficience intellectuelle ont donné lieu à des résultats contradictoires quant aux différences intersexes. La présente recherche compare les mères et les pères d'enfants ayant une déficience intellectuelle à l'égard de leur stress parental, de leur détresse, de leur bien-être et de leur habileté à résoudre des problèmes. L'échantillon comprend 111 mères et 88 pères ayant complété un questionnaire autoadministré. Les résultats montrent que les différences entre les parents tendent à disparaître lorsque des facteurs contextuels sont considérés. Toutefois, l'orientation négative à l'égard des problèmes se révèle une dimension importante dans l'adaptation des parents face au stress, car elle est reliée à leur santé psychologique. Il pourrait donc s'agir d'une cible d'intervention à privilégier.
\end{abstract}

\section{PROBLÉMATIQUE}

Maintes recherches démontrent que les parents d'enfants ayant une déficience intellectuelle sont plus enclins que ceux d'enfants tout-venant à rapporter du stress, de la détresse et de la dépression (Lachance, Richer, Côté et Poulin, 2004; Olsson et Hwang, 2001) ainsi qu'un moindre niveau de bienêtre (Emerson, Hatton, Llewellyn, Blacker et Graham, 2006). Afin de mieux comprendre leur réalité, plusieurs études ont examiné les caractéristiques des parents (sexe, scolarité, statut d'emploi) de même que celles de l'enfant (âge, sévérité de la déficience intellectuelle) et de la famille (monoparentalité, présence d'autres enfants au développement atypique) pouvant être liées au stress et à la santé psychologique des parents

Natasha Tremblay, psychologue, Centre de réadaptation en déficience intellectuelle et en troubles envahissants du développement (CRDITED) du Saguenay-Lac-Saint-Jean, département des sciences de la santé, Université du Québec à Chicoutimi (UQAC), adresse électronique : natasha.tremblay@ssss.gouv.qc.ca; Lise Lachance, professeure titulaire, département d'éducation et pédagogie, Université du Québec à Montréal ; Alain Côté, conseiller à la direction des services professionnels et de la recherche, CRDITED du Saguenay-Lac-Saint-Jean ; Louis Richer, professeur titulaire, département des sciences de la santé, UQAC.
(Conseil de la famille et de l'enfance, 2007; Emerson et al., 2006; Lachance et al., 2004; Olsson et Hwang, 2001). Les recherches menées pour comparer la détresse des mères et des pères ont donné lieu à des résultats contradictoires. Alors que des auteurs rapportent davantage de détresse et de dépression chez les mères (Lachance et al., 2004; Olsson et Hwang, 2001), d'autres ne relèvent pas de différence à l'égard de la détresse lorsque des caractéristiques du parent, de l'enfant et de la situation familiale sont contrôlées (Khamis, 2006; Lachance et al., 2004).

Les auteurs tiennent à remercier Karine N. Tremblay pour sa collaboration et à exprimer leur gratitude aux organismes suivants pour leur soutien financier: le Fonds de recherche sur la société et la culture et ses partenaires (Curateur public, ministère de la Justice, ministère de la Santé et des Services sociaux, ministère de l'Éducation, du Loisir et du Sport, ministère de l'Emploi et de la Solidarité sociale, ministère de la Sécurité publique, ministère des Transports, Office des personnes handicapées du Québec, Société d'habitation du Québec, Fonds de recherche en santé du Québec), le Consortium national de recherche sur l'intégration sociale ainsi que la Fondation de l'Université du Québec à Chicoutimi, la Fondation du CRDITED du Saguenay-Lac-StJean, la Fondation Asselin du Cégep de Jonquière, le Syndicat des professeures et professeurs de l'Université du Québec à Chicoutimi et, finalement, le Département des sciences de l'éducation et de psychologie de l'Université du Québec à Chicoutimi. 
À ce jour, les chercheurs peuvent difficilement expliquer les différences trouvées entre les parents sur le plan de la santé psychologique. Le modèle proposé par D'Zurilla et ses collaborateurs (D'Zurilla et Goldfried, 1971 ; D'Zurilla, Nezu et Maydeu-Olivares, 2002, 2004) sur la résolution de problèmes sociaux s'avère prometteur en ce sens, car il s'intéresse à l'habileté à résoudre des problèmes au quotidien (Chang, D'Zurilla et Sanna, 2004; D'Zurilla et Nezu, 1990). Ce modèle énonce que l'orientation d'une personne face à un problème peut être positive ou négative. L'orientation positive permet habituellement à une personne d'adopter un style de résolution de problèmes rationnel, alors que l'orientation négative l'amène généralement à recourir aux deux styles de résolution dysfonctionnels que sont l'impulsivité/négligence et l'évitement (D'Zurilla et al., 2004). Les composantes de ce modèle ont été corrélées significativement à plusieurs états psychologiques négatifs (dépression, anxiété) (D’Zurilla, Chang, Nottingham et Faccini, 1998; Gosselin, Dugas et Ladouceur, 2002; McMurran et Christopher, 2009) et positifs (bien-être, satisfaction de vie) (Chang, Downey et Salata, 2004; Chang et al., 2009; Goldberg-Arnold, 1998). Les résultats de ces recherches suggèrent, d'une part, que l'orientation positive et le style rationnel diminueraient la présence et l'intensité des états psychologiques négatifs, alors qu'ils produiraient l'effet contraire sur les états psychologiques positifs. D'autre part, l'orientation négative entraînerait un effet inverse en intensifiant les états psychologiques négatifs et en réduisant les états positifs.

Les recherches portant sur le modèle de résolution de problèmes sociaux ont aussi permis de mettre en évidence que la résolution de problèmes est un processus pouvant intervenir comme médiateur entre le stress ressenti et la santé psychologique (D'Zurilla et Nezu, 2007; Nezu, Nezu et D'Zurilla, 2010). Les événements de vie stressants déclencheraient la résolution de problèmes qui, à son tour, agirait sur l'adaptation de l'individu. Des études ont fait ressortir la présence d'effets médiateurs de la résolution de problèmes dans les relations entre les stresseurs quotidiens et les symptômes dépressifs et anxieux (Bell et D'Zurilla, 2009; Kant, D'Zurilla et Maydeu-Olivares, 1997) ainsi qu'entre le stress et le bien-être psychologique (Chang et al., 2009). Ces résultats appuient la conception voulant que l'habileté générale et chacune des composantes de la résolution de problèmes puissent jouer un rôle médiateur entre le stress et des indicateurs positifs ou négatifs de la santé psychologique.

D'Zurilla et Nezu (2007) ont proposé un programme d'intervention, la Thérapie de résolution de problèmes, ciblant plusieurs éléments clés dont les symptômes dépressifs, la gestion du stress et l'utilisation de stratégies d'ajustement inefficaces. Les auteurs rapportent une série d'études venant appuyer empiriquement l'efficacité de ce programme auprès de différentes populations cliniques, dont des parents d'enfants ayant des difficultés comportementales ou des problèmes de santé mentale ou physique. Ce programme peut être utilisé tant à des fins de prévention, que d'intervention ainsi que pour le maintien des stratégies acquises. Il devient alors pertinent de vérifier si l'habileté des parents d'enfants ayant une déficience intellectuelle à résoudre des problèmes peut médiatiser le lien entre le stress et des indicateurs négatifs et positifs de la santé psychologique afin d'évaluer la pertinence d'appliquer la Thérapie de résolution de problèmes auprès d'eux. Une compréhension mieux articulée du vécu parental pourrait vraisemblablement conduire à la mise en place d'interventions plus appropriées visant à réduire le stress et, conséquemment, à contrer l'émergence de problèmes de santé psychologique.

\section{OBJECTIFS}

Le premier objectif de cette étude est de comparer les mères et les pères d'enfants ayant une déficience intellectuelle à l'égard de la perception de leur stress parental, de leur santé psychologique et de leur habileté à résoudre des problèmes. Le second objectif est de vérifier si l'habileté des mères et des pères à résoudre des problèmes exerce un rôle médiateur entre le stress parental et deux indicateurs de santé psychologique, soit la détresse et le bienêtre.

\section{MÉTHODE}

\section{Déroulement}

L'ensemble des parents d'enfants d'âge scolaire recevant des services d'un centre de réadaptation en déficience intellectuelle et en troubles envahissants du développement a été contacté. Parmi ceux-ci, $63 \%$ ont accepté de répondre à domicile à un 
questionnaire auto-administré (environ 30 minutes). Les parents retenus sont ceux des enfants dont la déficience intellectuelle a pu être attestée par des résultats d'évaluation du fonctionnement cognitif et des comportements adaptatifs.

\section{$\underline{\text { Participants }}$}

L'échantillon comprend 111 mères et 88 pères. La plupart $(88 \%)$ sont les parents biologiques de l'enfant ayant une déficience intellectuelle et vivent en couple ( $86 \%)$. L'âge moyen des parents est de 43 ans $(E ́ T=7,6)$. Cependant, les mères sont significativement plus jeunes $(M=41,8$ ans; $E ́ T=7,5)$ que les pères $(M=44,5$ ans; $E ́ T=7,6)$ $[t(197)=-2,43, p<0,05]$. Les pères sont plus nombreux $(83 \%)$ que les mères $(54 \%)$ à être actifs sur le marché du travail $\left[\chi^{2}(1, N=199)=18,50\right.$, $p<0,001]$ et $85 \%$ des répondants considèrent leur revenu familial suffisant pour subvenir aux besoins de la famille. L'âge moyen des enfants ayant une déficience intellectuelle est de 13,4 ans $(E ́ T=4,9)$ et plus de la moitié sont des garçons (52\%). Le niveau de déficience intellectuelle des enfants se répartit comme suit : léger (15\%), moyen (41\%), grave $(29 \%)$ et profond $(15 \%)$, alors que le niveau de déficit des comportements adaptatifs est léger pour près du tiers des enfants $(32 \%)$.

\section{$\underline{\text { Instruments }}$}

Les parents ont fourni des renseignements sociodémographiques et ont répondu à des instruments ayant déjà fait l'objet d'une validation en français.

\section{Stress parental}

Issue de l'Indice de stress parental (Bigras, Lafrenière et Abidin, 1996), l'Échelle de restriction dans le rôle parental évalue l'effet négatif de la parentalité sur la liberté personnelle. Les répondants doivent indiquer leur niveau d'accord pour sept items à l'aide d'une échelle de type Likert (de 1 = fortement en désaccord à 5 = fortement en accord). Selon les normes disponibles, un score de 26 et plus correspond à un niveau élevé de restrictions parentales. Le coefficient alpha du présent échantillon est de 0,83 .

\section{Détresse psychologique}

La version abrégée de l'Indice de détresse psychologique (IDPESQ; Préville, Boyer, Potvin, Perrault et Légaré, 1992) évalue la fréquence (de 1 = jamais à 4 = très souvent) de 14 symptômes liés à la détresse en se référant aux 7 derniers jours. Des normes, en fonction du sexe et de l'âge $\left(85^{\mathrm{e}}\right.$ percentile), sont disponibles pour la population québécoise (Boyer, Préville, Légaré et Valois, 1993). Le coefficient alpha de la présente étude est de 0,91 .

\section{Bien-être}

L'Échelle de Mesure des Manifestations du Bienêtre Psychologique (EMMBEP; Massé et al., 1998) inclut 25 items qui permettent au répondant de faire une évaluation de ses réalisations, du contrôle de soi et des événements, de son équilibre psychologique, de sa sociabilité et de son niveau de bonheur à partir d'une échelle de type Likert (de $1=$ jamais à $5=$ presque toujours). Un résultat au-dessus de 96 (quintile supérieur) sert à établir un niveau de bienêtre élevé (Institut de la statistique du Québec-ISQ, 2010). Le coefficient alpha du présent échantillon s'élève à 0,94 .

\section{Résolution de problèmes sociaux}

L'adaptation francophone (Gosselin et al., 2002) de la version abrégée (25 items) de l'Inventaire de résolution de problèmes sociaux révisé (SPSI-R-S ; D'Zurilla et al., 2002) évalue l'habileté à résoudre des problèmes à l'aide d'une échelle en cinq points (de 1 = pas du tout vrai à $5=$ extrêmement vrai). Elle permet de dégager un score global et un score pour chacune des 5 dimensions du modèle de résolution de problèmes sociaux: les orientations positive et négative ainsi que les styles rationnels, impulsivité/négligence et évitement. Des normes, en fonction de l'âge, permettent d'établir si un répondant fait preuve de difficultés face à la résolution de problèmes. Les coefficients alpha de la présente étude varient de 0,67 à 0,78 , sauf celui du style évitement qui est plus faible $(0,56)$.

\section{RÉSULTATS}

\section{Analyses descriptives}

Les résultats montrent qu'environ $19 \%$ des parents rapportent un niveau élevé de restrictions dans leur rôle parental comparativement au $15 \%$ attendu dans la population générale (Bigras et al., 1996). Plus de $42 \%$ des participants rapportent un niveau élevé de détresse tandis que $3 \%$ présentent un fort niveau de bien-être. Des taux respectifs de $23 \%$ et de $19 \%$ ont été observés pour ces mêmes variables au sein 
de la population québécoise (ISQ, 2010). Enfin, $46 \%$ des parents présentent des difficultés en matière de résolution de problèmes, alors qu'un taux de $16 \%$ est attendu (D’Zurilla et al., 2002).

\section{Comparaisons entre les mères et les pères}

Dans un premier temps, des tests $t$ pour échantillons indépendants ont servi à comparer les mères et les pères à l'égard de la perception de leur stress parental, de leur santé psychologique et de leur habileté à résoudre des problèmes (objectif 1) (voir Tableau 1). Les résultats indiquent que les mères obtiennent des moyennes plus élevées que les pères concernant le stress parental et la détresse. Toutefois, aucune différence significative n'est relevée entre les parents concernant le bien-être. Quant à la résolution de problèmes, une seule différence s'avère significative. Les mères présentent des scores moyens supérieurs à ceux des pères quant à l'orientation négative face aux problèmes.
Une deuxième série d'analyses a été effectuée afin de contrôler des variables liées aux caractéristiques et au contexte de vie des parents. Il s'agit de l'occupation d'un emploi, du dernier niveau de scolarité complété et de leur situation familiale puisque ces trois variables entretiennent des liens significatifs avec le stress parental, la santé psychologique ou la résolution de problèmes. L'âge des parents a également été contrôlé en raison de la différence significative relevée entre les mères et les pères de l'échantillon. Une analyse de régression hiérarchique a donc été appliquée pour chacune des variables à l'étude. Le premier bloc comprend les variables contrôles, alors que le second inclut le sexe du parent. Suivant ces analyses, une seule différence entre les mères et les pères est significative (voir Tableau 2). Les mères présentent un style moins évitant que les pères $(\beta=0,17, p<0,05)$. Toutefois, la contribution unique de cet effet ne s'élève qu'à $2 \%$.

\section{$\underline{\text { Tableau } 1}$}

\section{Moyennes, écarts-types et comparaison des variables à l'étude en fonction du sexe du parent}

\begin{tabular}{|c|c|c|c|c|c|c|c|c|c|c|c|}
\hline \multirow{2}{*}{ Variables } & \multicolumn{3}{|c|}{ Total $(n=199)$} & \multicolumn{3}{|c|}{ Mères $(n=111)$} & \multicolumn{3}{|c|}{ Pères $(n=88)$} & \multirow{2}{*}{\multicolumn{2}{|c|}{ Test $t$}} \\
\hline & $N$ & $M$ & $E ́ T$ & $N$ & $M$ & 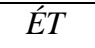 & $N$ & $M$ & 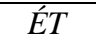 & & \\
\hline Stress parental & 199 & 21,00 & 6,52 & 111 & 22,04 & 6,37 & 88 & 19,70 & 6,51 & 2,54 & $*$ \\
\hline Détresse & 199 & 25,24 & 17,66 & 111 & 27,69 & 19,50 & 88 & 22,14 & 14,56 & 2,22 & $*$ \\
\hline Bien-être & 199 & 71,07 & 14,03 & 111 & 69,44 & 15,12 & 88 & 73,11 & 12,30 & $-1,84$ & \\
\hline \multicolumn{12}{|l|}{ Résolution de problèmes } \\
\hline Score global & 189 & 10,47 & 1,93 & 104 & 10,41 & 2,03 & 85 & 10,53 & 1,81 & $-0,43$ & \\
\hline Orientation positive & 198 & 2,32 & 0,75 & 111 & 2,27 & 0,78 & 87 & 2,38 & 0,71 & $-1,00$ & \\
\hline Orientation négative & 196 & 1,97 & 0,64 & 108 & 1,86 & 0,64 & 88 & 2,10 & 0,61 & 2,73 & $* *$ \\
\hline Style rationnel & 198 & 2,06 & 0,75 & 111 & 2,02 & 0,77 & 87 & 2,10 & 0,72 & $-0,72$ & \\
\hline Style impulsivité/négligence & 192 & 2,00 & 0,61 & 105 & 2,05 & 0,62 & 87 & 1,94 & 0,60 & 1,21 & \\
\hline Style évitement & 196 & 2,26 & 0,65 & 110 & 2,32 & 0,64 & 86 & 2,18 & 0,65 & 1,50 & \\
\hline
\end{tabular}

${ }^{*} p<0,05 . * * p<0,01$. 


\section{$\underline{\text { Effet médiateur de la résolution de problèmes }}$}

La vérification des effets médiateurs de la résolution de problèmes entre le stress parental et la santé psychologique des mères et des pères (objectif 2) s'appuie sur la procédure de Baron et Kenny (1986). Ces auteurs énoncent trois critères à respecter pour qu'un effet soit considéré comme médiateur. Tout d'abord, la variable indépendante (stress parental) doit être reliée à la variable médiatrice (résolution de problèmes) (critère 1). Deuxièmement, la variable médiatrice doit être associée à la variable dépendante (détresse, bien-être) (critère 2). Troisièmement, la variable indépendante doit être liée à la variable dépendante (critère 3). De plus, pour qualifier un effet de médiateur, la relation entre la variable indépendante et la variable dépendante doit être modifiée significativement lorsque la variable médiatrice est prise en considération. À cet effet, le test de Sobel a été retenu. Lorsque la relation entre la variable indépendante et dépendante n'est plus significative, il s'agit d'un effet médiateur complet alors que si cette relation diminue, mais demeure significative, l'effet est considéré comme partiel.

Suivant l'examen de la matrice de corrélations partielles (voir Tableau 3), il apparaît que le stress parental est lié à l'orientation négative (critère 1) laquelle est associée à la détresse et au bien-être (critère 2), et ce, indépendamment du sexe du parent. Dans le cas des pères, le stress parental est aussi relié au score global de résolution de problèmes (critère 1), mais ce dernier n'est pas lié à leur santé psychologique. Enfin, le stress parental est lié à la détresse et au bien-être des mères et des pères (critère 3 ). Bref, considérant les trois critères à respecter, seule l'orientation négative est susceptible d'exercer un rôle médiateur entre le stress parental et les deux indicateurs de santé psychologique chez les parents. Des analyses de régression hiérarchique distinctes ont donc été réalisées pour les mères et les pères. Il en résulte que l'orientation négative intervient entre le stress et la détresse seulement pour les mères $(Z$ de Sobel $=3,10, p<0,05)$. Étant donné que la relation entre le stress et la détresse demeure significative, cet effet médiateur est partiel.

\section{DISCUSSION}

Cette étude visait à comparer la réalité des mères et des pères à l'égard du stress, de la santé psychologique et de la résolution de problèmes. Les résultats montrent que les mères et les pères ne diffèrent pas de façon significative sur le stress et la détresse lorsque l'âge, le statut d'emploi, la scolarité et la situation familiale sont considérés. Selon ces résultats, les pères sont aussi à risque que les mères de rapporter des problèmes de santé psychologique. La mise en place des services pour les parents devrait donc tenir compte tant des pères que des mères. Puisque les mères demandent davantage d'aide que les pères en regard des difficultés qu'elles rencontrent (Saloviita, Itälinna et Leinonen, 2003), il importe de se questionner sur la façon d'intervenir pour s'assurer de rejoindre les pères.

Cette étude a également permis de mettre en lumière des facteurs pouvant rendre les parents plus vulnérables, dont la monoparentalité, l'absence d'un emploi rémunéré ainsi qu'un niveau de scolarité moins élevé. L'occurrence de l'un ou de plusieurs de ces facteurs pourrait ainsi permettre de prioriser les parents davantage susceptibles d'avoir besoin de services psychosociaux. Ainsi, les praticiens sociaux, et particulièrement ceux dispensant des services spécifiques de première ligne en déficience intellectuelle, devraient rehausser leur vigilance en regard des parents monoparentaux puisque ceux-ci présentent un risque accru d'éprouver de la détresse. En lien avec l'occupation d'un emploi, une étude précédente (Lachance et al., 2004) avait démontré que certains parents se retiraient du marché du travail en raison de problèmes de conciliation travail-famille, et ce, nonobstant le fait que leur emploi pouvait se révéler autant une source de répit que de valorisation. La présente étude montre, en outre, que les parents qui occupent un emploi et qui sont plus scolarisés présentent une meilleure habileté de résolution de problèmes et se montrent moins évitants face aux problèmes auxquels ils sont confrontés sur une base quotidienne. Les politiques de l'État, et notamment les orientations du réseau de la santé et des services sociaux, devraient prendre en compte cet aspect afin d'amener les prestateurs de services à mieux soutenir les mères et les pères d'enfants ayant des déficiences dans l'accomplissement des rôles qu'ils choisissent d'assumer.

Pour ce qui est de la comparaison de l'habileté à résoudre des problèmes en fonction du sexe du parent, une seule différence significative a été observée lorsque les variables contextuelles sont considérées. En effet, les mères présentent un style moins évitant que les pères en regard du problème à 
résoudre. Il se pourrait que l'investissement des qu'elles utilisent plus de stratégies et qu'elles se sentent plus compétentes (Grant et Whittell, 2000), puisse mener les pères à adopter un style évitant face aux problèmes. Toutefois, il est possible de soulever la question à savoir si les mères s'impliquent davantage en réponse à l'évitement des pères ou si ce n'est pas plutôt la prise en charge des responsabilités familiales par les mères qui amène les pères à devenir plus évitants. En outre, ces derniers pourraient avoir davantage tendance à recourir au soutien conjugal lors de la résolution des situations problématiques (Saloviita et al., 2003). Cette étude visait aussi à vérifier si l'habileté à résoudre des problèmes pouvait avoir un effet médiateur dans la relation entre le stress et les indicateurs de santé psychologique. Seul un effet médiateur a été identifié chez les mères: une orientation négative moins élevée est associée à une diminution de l'intensité du lien entre le stress parental et la détresse. Même si cet effet médiateur n'est pas observé chez les pères, il faut noter qu'un plus haut niveau de stress parental est relié à une orientation négative plus élevée, peu importe le sexe. Les résultats de la présente étude ont également montré que plus les parents s'orientent négativement face aux problèmes, plus ils rapportent de détresse et moins ils ressentent de bien-être. Cependant, le stress des parents n'est pas lié à l'orientation positive. Le stress des pères est associé à une moins bonne habileté à résoudre des problèmes, alors que ce lien n'est pas démontré chez les mères. En somme, concernant la résolution de problèmes, c'est l'adoption d'une orientation négative qui semble faire une différence quant au niveau de détresse ressentie par les parents d'enfants ayant une déficience intellectuelle. Les parents se trouvant dans un contexte de vie difficile, et particulièrement ceux rencontrant les facteurs de risque précédemment identifiés, auraient avantage à recevoir de l'aide pour changer leur façon de percevoir les problèmes auxquels ils se trouvent confrontés.

L'intervention auprès de ces parents pourrait dès lors se concentrer sur la modification de l'orientation négative. En ce sens, D'Zurilla et Nezu (2007) précisent que le programme d'intervention sur la résolution de problèmes peut être adapté à des mères dans les soins donnés aux enfants, par le fait besoins spécifiques. Ainsi, chez les parents d'enfants ayant une déficience intellectuelle, le choix des modules du programme à utiliser pourrait se centrer sur la modification de l'orientation négative afin d'en arriver à une meilleure efficience des services. Par exemple, l'adoption d'un style évitement chez les pères pourrait devenir une cible d'action pertinente, et ce, tant en termes de prévention que d'intervention. En effet, de telles interventions pourraient contribuer à prévenir le développement de la détresse. En devenant de moins en moins évitants face aux problèmes auxquels ils sont confrontés, certains pères pourraient être amenés à s'impliquer davantage dans la répartition des tâches au sein du couple. Une approche systémique d'intervention pourrait être privilégiée puisqu'il est possible que l'interaction des pères avec leur conjointe influence leur choix d'adopter un style de résolution de problèmes en particulier. Étant donné que D'Zurilla et Nezu (2007) proposent de développer des interventions adaptées de la thérapie cognitive visant à modifier les distorsions cognitives qui pourraient être présentes à propos des croyances et des pensées, il importe de se questionner sur le type d'intervenants psychosociaux pouvant assumer ce genre d'intervention.

Cette étude s'est intéressée à des indicateurs de santé psychologique des parents, mais n'a pas abordé directement des problèmes de santé mentale, comme la dépression. Il va de soi que l'intensité relative du malaise psychique vécu par les parents devrait, tôt ou tard, alimenter une réflexion plus approfondie sur les services dont ceux-ci auraient légitimement besoin. Ainsi, l'intervention pourrait être différente en fonction de la gravité de la problématique identifiée. Les modalités d'intervention devraient, somme toute, s'adapter en fonction de l'état du parent. Par exemple, une approche en groupe pourrait ne pas se révéler suffisante, voire contre-indiquée pour des parents présentant un niveau élevé de détresse. L'efficacité des interventions psychosociales dans ce domaine devrait aussi faire l'objet de travaux de recherche afin de vérifier si le modèle d'intervention proposé peut s'appliquer de la même façon à tous les parents. 
$\underline{\text { Tableau } 2}$

Analyses de régression hiérarchique pour les différences intersexes

\begin{tabular}{|c|c|c|c|c|c|c|c|c|c|c|c|c|c|c|c|c|c|c|c|}
\hline & \multicolumn{2}{|c|}{ STRESP } & \multicolumn{2}{|c|}{ SPSI } & \multicolumn{2}{|c|}{ PPO } & \multicolumn{2}{|c|}{ NPO } & \multicolumn{3}{|c|}{ RPS } & \multicolumn{2}{|c|}{ ICS } & \multicolumn{2}{|c|}{ AS } & \multicolumn{2}{|c|}{ DETRES } & \multicolumn{2}{|c|}{ BIENETR } \\
\hline & $\beta$ & $S r^{2}$ & $\beta$ & $S r^{2}$ & $\beta$ & $S r^{2}$ & $\beta$ & $S r^{2}$ & $\beta$ & & $S r^{2}$ & $\beta$ & $S r^{2}$ & $\beta$ & $S r^{2}$ & $\beta$ & $S r^{2}$ & $\beta$ & $S r^{2}$ \\
\hline \multicolumn{20}{|l|}{ Bloc 1} \\
\hline Âge du parent & 0,03 & & 0,09 & & $-0,09$ & & $-0,10$ & & 0,06 & & & $-0,17^{*}$ & 0,02 & $-0,16^{*}$ & 0,02 & 0,00 & & $-0,02$ & \\
\hline Emploi & $-0,15$ & & $0,21^{* *}$ & 0,04 & 0,12 & & $-0,11$ & & 0,07 & & & $-0,11$ & & $-0,19^{*}$ & 0,03 & $-0,04$ & & 0,07 & \\
\hline Scolarité & 0,12 & & $0,26^{* * *}$ & 0,07 & $0,15^{*}$ & 0,02 & $-0,14$ & & 0,21 & $* *$ & 0,04 & $-0,19^{* *}$ & 0,04 & $-0,20^{* *}$ & 0,04 & 0,06 & & $-0,11$ & \\
\hline $\begin{array}{l}\text { Situation } \\
\text { familiale }\end{array}$ & 0,06 & & 0,08 & & $0,23^{* *}$ & 0,05 & 0,14 & & 0,18 & $* *$ & 0,03 & 0,05 & & 0,02 & & $0,26^{* * * *}$ & 0,06 & 0,02 & \\
\hline \multicolumn{20}{|l|}{ Bloc 2} \\
\hline Sexe du parent & $-0,12$ & & $-0,02$ & & 0,08 & & $-0,14$ & & 0,06 & & & 0,13 & & $0,17^{*}$ & 0,02 & $-0,12$ & & 0,11 & \\
\hline$R^{2}$ & 0,07 & & 0,13 & & 0,11 & & 0,09 & & 0,10 & & & 0,08 & & 0,10 & & 0,10 & & 0,03 & \\
\hline$R^{2}$ ajusté & 0,04 & & 0,11 & & 0,08 & & 0,06 & & 0,07 & & & 0,05 & & 0,08 & & 0,07 & & 0,01 & \\
\hline
\end{tabular}

Note. STRESP $=$ Stress parental. SPSI = Score global de la résolution de problèmes. $\mathrm{PPO}=$ Orientation positive. $\mathrm{NPO}=$ Orientation négative. RPS = Style rationnel. ICS = Style impulsivité/négligence. AS = Style évitement. DETRES $=$ Détresse. BIENETR $=$ Bien-être.

$* p<0,05 . * * p<0,01 . * * * p<0,001$. 


\section{$\underline{\text { Tableau } 3}$}

Matrice de corrélations partielles entre les variables à l'étude après avoir contrôlé l'âge, le niveau de scolarité,

l'occupation d'un emploi et la monoparentalité

\begin{tabular}{|c|c|c|c|c|c|c|c|c|c|c|c|c|}
\hline & Sexe & STRESP & & SPSI & PPO & NPO & RPS & ICS & AS & DETRES & BIENETR & \\
\hline STRESP & $-0,11$ & 1 & & $-0,05$ & 0,06 & $-0,35^{* * *}$ & 0,09 & $-0,11$ & $-0,11$ & $0,53^{* * *}$ & $-0,34^{*}$ & **** \\
\hline SPSI & 0,01 & $-0,30$ & $* *$ & 1 & $0,63^{* * * *}$ & $0,51^{* * *}$ & $0,55^{* * * *}$ & $0,57^{* * *}$ & $0,60^{* * *}$ & $-0,28^{* *}$ & 0,24 * & * \\
\hline PPO & 0,06 & $-0,21$ & & $0,60^{* * *}$ & 1 & $-0,11$ & $0,59^{* * *}$ & $-0,07$ & 0,11 & 0,11 & 0,16 & \\
\hline NPO & $0,16^{*}$ & $-0,25$ & * & $0,49^{* * *}$ & 0,09 & 1 & $-0,18$ & $0,42^{* * *}$ & $0,46^{* * *}$ & $-0,51^{* * *}$ & $0,26^{*}$ & ** \\
\hline RPS & 0,07 & $-0,11$ & & $0,52^{* * *}$ & $0,51^{* * *}$ & $-0,16$ & 1 & 0,05 & 0,03 & 0,13 & 0,01 & \\
\hline ICS & $-0,09$ & $-0,15$ & & $0,60^{* * *}$ & $-0,04$ & $0,26^{*}$ & 0,03 & 1 & $0,37^{* * *}$ & $-0,38^{* * *}$ & $0,21 *$ & * \\
\hline AS & $-0,13$ & $-0,19$ & & $0,64^{* * *}$ & 0,13 & $0,26^{*}$ & 0,00 & $0,64^{* * *}$ & 1 & $-0,28^{* *}$ & 0,07 & \\
\hline DETRES & $-0,11$ & 0,35 & $* * *$ & $-0,20$ & $-0,15$ & $-0,28^{* *}$ & $-0,02$ & $-0,10$ & $-0,06$ & 1 & $-0,48$ * & **** \\
\hline BIENETR & 0,09 & $-0,28$ & $*$ & 0,14 & 0,16 & $0,25^{*}$ & 0,12 & $-0,15$ & $-0,06$ & $-0,30^{* *}$ & 1 & \\
\hline
\end{tabular}

Note . Triangle inférieur $=$ pères; Triangle supérieur $=$ mères. STRESP $=$ Stress parental. SPSI $=$ Score global de la résolution de problèmes. $\mathrm{PPO}=$ Orientation positive .

$\mathrm{NPO}=$ Orientation négative. $\mathrm{RPS}=$ Style rationnel. ICS $=$ Style impulsivité/négligence. AS = Style évitement. DETRES = Détresse. BIENETR = Bien-être.

$* p<0,05 . * * p<0,01 . * * * p<0,001$ 


\section{CONCLUSION}

Les résultats de cette étude démontrent qu'il est nécessaire de revoir la façon d'aborder le stress et la détresse des parents d'enfants ayant une déficience intellectuelle. Ainsi, au moment où l'organisation des services spécialisés tend à se mettre en place dans le domaine de la déficience intellectuelle, les résultats obtenus font ressortir l'importance de se questionner sur la prise en charge des parents d'enfants ayant une déficience intellectuelle afin d'éviter que ceux-ci se retrouvent face à un vide de services. Alors que les services spécifiques qui ont cours en première ligne tendent à offrir un soutien plutôt ponctuel, l'état psychologique de plusieurs parents nécessiterait une intervention plus complexe qui relèverait d'intervenants de première ligne spécialisés ou encore de services à dispenser par la seconde ligne. L'évaluation de l'état psychologique des parents à l'aide d'outils validés pourrait permettre de diriger les parents vers des services plus adaptés à leurs besoins.

\title{
PROBLEM-SOLVING ABILITY OF PARENTS HAVING CHILDREN WITH AN INTELLECTUAL DISABILITY
}

\begin{abstract}
Studies on stress and psychological health of parents having children with an intellectual disability have reported inconsistent results concerning sex differences. The present research compares mothers and fathers of children with an intellectual disability regarding parental experience of stress, distress, wellbeing and problem-solving abilities. The sample included 111 mothers and 88 fathers who completed a self-report questionnaire. Results show that differences between parents tend to disappear when contextual factors are considered. However, the negative orientation towards problems represents an important dimension in the adaptation of parents dealing with stress as it relates to their psychological health. This could therefore be an option to consider for intervention.
\end{abstract}

\section{BIBLIOGRAPHIE}

BARON, R. M., KENNY, D. A. (1986). The moderator-mediator variable distinction in social psychological research : Conceptual, strategic, and statistical considerations. Journal of Personality \& Social Psychology, 51, 1173-1182.

BELL, A. C., D'ZURILlA, T. J. (2009). The influence of social problem-solving ability on the relationship between daily stress and adjustment. Cognitive Therapy Research, $33,439-448$.

BIGRAS, M., LAFRENIÈRE, P. J., ABIDIN, R. R. (1996). Indice de stress parental: Manuel francophone en complément de l'édition américaine. Toronto : Multi-Health Systems.

BOYER, R., PRÉVILLE, M., LÉGARÉ, G., VALOIS, P. (1993). La détresse psychologique dans la population du Québec non institutionnalisée : résultats normatifs de l'enquête Santé Québec. Revue canadienne de psychiatrie, 38, 339-343.
CHANG, E. C., DOWNEY, C. A., SALATA, J. L. (2004). Social problem solving and positive psychological functioning: Looking at the positive side of problem solving. In E. C. Chang, T. J. D'Zurilla, \& L. J. Sanna. Social problem solving: Theory, research, and training. Washington: American Psychological Association.

CHANG, E. C., D'ZURILLA, T. J., SANNA, L. J. (2004). Social problem solving: Theory, research, and training. Washington: American Psychological Association.

CHANG, E. C., D'ZURILLA, T. J., SANNA, L. J. (2009). Social problem solving as a mediator of the link between stress and psychological well-being in middleadulthood. Cognitive Therapy Research, 33, 33-49.

CONSEIL DE LA FAMILLE ET DE L'ENFANCE. (2007). Tricoter avec amour. Étude sur la vie de famille avec un enfant handicapé. Québec: Gouvernement du Québec. 
D'ZURILLA, T. J., CHANG, E. C., NOTTINGHAM, E. J., FACCINI, L. (1998). Social problem-solving deficits and hopelessness, depression, and suicidal risk in college students and psychiatric inpatients. Journal of Clinical Psychology, 54, 1-17.

D'ZURILLA, T. J., GOLDFRIED, M. R. (1971). Problem solving and behaviour modification. Journal of Abnormal Psychology, 78, 107-126.

D'ZURILlA, T. J., NEZU, A. M. (1990). Development and preliminary evaluation of the social problem-solving inventory. Psychological Assessment: A Journal of Consulting and Clinical Psychology, 2, 156163.

D'ZURILlA, T. J., NEZU, A. M. (2007). ProblemSolving Therapy. A Positive Approach to Clinical Intervention. NewYork: Springer Publishing Company.

D'ZURILLA, T. J., NEZU, A M., MAYDEUOLIVARES, A. (2002). Social problemsolving inventory-revised (SPSI-R) manual. New York : Multi-Health Systems.

D'ZURILlA, T. J., NEZU, A. M., MAYDEUOLIVARES, A. (2004). Social problem solving: Theory and assessment. In E. C. Chang, T. J. D'Zurilla, \& L. J. Sanna. Social problem solving: Theory, research, and training. Washington: American Psychological Association.

EMERSON, E., HATTON, C. LLEWELLYN, G., BLACKER, J., GRAHAM, H. (2006). Socio-economic position, household composition, health status and indicators of the well-being of mothers of children with and without intellectual disabilities. Journal of Intellectual Disability Research, 50, 862873.

GOLDBERG-ARNOLD, J. S. (1998). Family problem solving : A resource and potential target for intervention in families of children with Down syndrome. Thèse de doctorat inédite, Binghamton University.

GOSSELIN, P., DUGAS, M. J., LADOUCEUR, R. (2002). Inquiétude et résolution de problèmes sociaux: le rôle de l'attitude négative face au problème. Journal de thérapie comportementale et cognitive, 12, 49-58.

GRANT, G., WHITTELL, B. (2000). Differentiated coping strategies in families with children or adults with intellectual disabilities: The relevance of gender, family, composition and the life span. Journal of Applied Research in Intellectual Disabilities, 13, 256-275.

INSTITUT DE LA STATISTIQUE DU QUÉBEC. (2010). Santé mentale et bien-être des adultes québécois : un aperçu à partir de quelques indicateurs-clés. Québec : Auteur.

KANT, G. L., D'ZURILLA, T. J., MAYDEUOLIVARES, A. (1997). Social problem solving as a mediator of stress-related depression and anxiety in middle-aged and elderly community residents. Cognitive Therapy \& Research, 21, 73-96.

KHAMIS, V. (2006). Psychological distress among parents of children with mental retardation in the United Arab Emirates. Social Science \& Medicine, 64, 850-857.

LACHANCE, L., RICHER, L., CÔTÉ, A., POULIN, J.-R. (2004). Conciliation travailfamille chez des parents d'enfants ou d'adolescents ayant une déficience intellectuelle. Chicoutimi : Université du Québec à Chicoutimi.

MASSÉ, R., POULIN, C., DASSA, C., LAMBERT, J., BÉLAIR, S., BATTAGLINI, M. A. (1998). Élaboration et validation d'un outil de mesure du bienêtre psychologique: l'ÉMMBEP. Revue canadienne de santé publique, 89, 352-357.

MCMURRAN, M., CHRISTOPHER, G. (2009). Social problem solving, anxiety, and depression in adult male prisoners. Legal \& Criminological Psychology, 14, 101-107.

NEZU, A. M., NEZU, C. M., D'ZURILLA, T. (2010). Problem-Solving Therapy. In N. Kazantzis, M. A. Reinecke, \& A. Freeman. Cognitive and Behavioral Theories in Clinical Practice. New York: The Guilford Press. 
OLSSON, M. B., HWANG, C. P. (2001). Depression in mothers and fathers of children with intellectual disability. Journal of Intellectual Disability Research, 45, 535543.

PRÉVILLE, M., BOYER, R., POTVIN, L, PERRAULT, C., LÉGARÉ, G. (1992). La détresse psychologique: détermination de la fiabilité et de la validité de la mesure utilisée dans l'enquête Santé Québec. Québec: Ministère de la Santé et des Services sociaux, Gouvernement du Québec.

SALOVIITA, M., ITÄLINNA, M., LEINONEN, E. (2003). Explaining the parental stress of fathers and mothers caring for a child with intellectual disability: A double ABCX model. Journal of Intellectual Disability Research, 47, 300-312. 